\title{
Vibrating wire for beam profile scanning
}

\author{
S. G. Arutunian, N. M. Dobrovolski, M. R. Mailian, I. G. Sinenko, and I.E. Vasiniuk \\ Yerevan Physics Institute, Alikhanian Brothers Street 2, 375036, Yerevan, Armenia
}

(Received 4 March 1999; published 9 December 1999)

\begin{abstract}
A method that measures the transverse profile (emittance) of the bunch by detecting radiation arising at the scattering of the bunch on scanning wire is widely used. In this work information about bunch scattering is obtained by measuring the oscillation frequency of the tightened scanning wire. In such a way, the system of radiation (or secondary particles) extraction and measurement can be removed. The entire unit consists of a compact fork with tightened wire and a scanning system. Normal oscillation frequency of a wire depends on wire tension, its geometric parameters, and, in a second approximation, its elastic characteristics. Normal oscillations are generated by interaction of an alternating current through the wire with magnetic field of a permanent magnet. In this case, it is suggested that the magnetic field of the accelerator (field of dipole magnets or quadrupole magnets) be used for excitation of oscillations. The dependence of oscillation frequency on beam scattering is determined by several factors, including changes of wire tension caused by transverse force of the beam and influence of beam self-field. Preliminary calculations show that the influence of wire heating will dominate. We have studied strain gauges on the basis of vibrating wire from various materials (tungsten, beryl bronze, and niobium zirconium alloys). A scheme of normal oscillation generation by alternating current in autogeneration circuit with automatic frequency adjustment was selected. A special method of wire fixation and elimination of transverse degrees of freedom allows us to achieve relative stability better than $10^{-5}$ during several days at a relative resolution of $10^{-6}$. Experimental results and estimates of wire heating of existing scanners show that the wire heats up to a few hundred grades, which is enough for measurements. The usage of wire of micrometer thickness diminishes the problem of wire thermalization speed during the scanning of the bunch.
\end{abstract}

PACS numbers: 29.27.Fh

\section{INTRODUCTION}

The method of beam transverse profile measurement in accelerators by scanning wire is widespread in accelerator technology [1-7]. In such a way, measurement of transverse profiles of proton, antiproton, ion, electron, and positron beams in a wide range of energies is carried out without destruction of the beam [1,3]. This method will also be used in new extra high energy accelerators (see, e.g., [7-9]).

The essence of the method is measurement of radiation and/or secondary particles arising at the scattering of the beam on scattering wire. Thus, the device consists of two essential units: a scanning mechanism with the wire fixed on a fork and a system of radiation (particles) detectors, located along the beam propagation.

In this work, we propose that a wire vibrating at its normal frequency may be used as a wire scanner. Note that pickups on the basis of vibrating wire are also widespread in technology and are used, in particular, in tensometry, gravimetry, measurements of magnetic field, and magnetic properties of materials (see, e.g., [10-12]).

A beam of charged particles affects vibrating wire frequency in several ways, namely, mechanical transverse influence caused by transferred pulse of the beam, influence of beam magnetic field, radiation effect of the beam on the wire material, and heating of the wire. The last effect dominates since, for typical currents of accelerator beams, the wire of micrometer diameter is heated over $1000 \mathrm{~K}[3,4]$.
In this work the strong dependence of normal frequency on its temperature and, hence, on the beam scattering is proposed to be used for measurement of beam local intensity. The indubitable advantage of this method is the compactness of the whole system and the elimination of the unit of radiation detectors, which leads to the reduction of the price.

The authors of the present work have accumulated a certain experience in development and production of tension gauges on the basis of vibrating wire. In particular, good results on frequency long-range relative stability $\sim 10^{-5}$ at relative resolution $\sim 10^{-6}$ are obtained. Such pickups were used for the measurement of spatial distribution of the magnetic field $[13,14]$.

Below we describe the main processes taking place at beam scattering on the wire. Results of the wire heating modeling are also presented, and specific problems arising at the development of vibrating wire scanners are discussed.

\section{EQUILIBRIUM TEMPERATURE AND WIRE THERMALIZATION}

Consider a long thin round wire, strained along the $y$ axis in vacuum. Wire ends are kept at constant temperature $T_{0}$. "Thin" and "long" mean that the wire diameter $d$, its length $l$, and part of the wire irradiated by the beam $\sigma_{y}$ satisfy the condition $d \ll \sigma_{y} \leq l$. The 
beam propagates along the $z$ axis and the wire scans the beam in the transverse direction along the $x$ axis.

Equilibrium temperature of an immobile wire in vacuum under the beam is defined by the balance between the power $\Delta E / \Delta t$ brought to it by the beam and two factors of heat removal: thermal radiation through the wire surface $W_{1}$ and thermoconductivity of the wire material through its ends $W_{2}$.

The power released on the wire can be estimated by the formula

$$
\frac{\Delta E}{\Delta t}=k\left(\frac{d E}{d z} d\right)\left(p(x) \sigma_{y} d\right),
$$

where $d E / d z$ is the loss of the beam particles during ionization in wire material, $p(x)$ is the local flux density of the beam particles, and $k$ is the coefficient characterizing the part of the scattered beam energy working on the wire heating.

The formula (1) has a simple meaning: the expression in the first pair of the parentheses defines the losses of a particle when it passes through the wire material, the second member defines the number of scattered particles in a unit of time. In Refs. [3,4], $k$ is set equal to $1 / 3$.

The thermal radiation $W_{1}$ is determined by the formula

$$
W_{1}=S_{\text {side }} \sigma\left(T^{4}-T_{0}^{4}\right),
$$

where $S_{\text {side }}$ is the side surface of the wire $\left(S_{\text {side }}=\pi d \sigma_{y}\right)$, $\sigma$ is the constant of Stefan-Boltzmann, and $T$ is the absolute temperature of the wire.

Heat loss through the wire ends is approximately equal

$$
W_{2}=S \frac{2 K}{l}\left(T-T_{0}\right)
$$

where $S=\pi d^{2} / 4$ is the wire cross section and $K$ is the thermal conductivity of wire material, which depends on temperature.

Figure 1 shows calculated curves $W_{1}, W_{2}$, and $W_{1}+W_{2}$ for tungsten wires of length $l=40 \mathrm{~mm}$ and diameters $d_{1}=10 \mu \mathrm{m}$ and $d_{2}=30 \mu \mathrm{m}\left(\sigma_{y}=1 \mathrm{~mm}\right)$. One can see that at the same absorption power the thin wire is heated more than the thick one, and this difference rises with increment of power. Presented curves show that to heat the wire of diameter $30 \mu \mathrm{m}$ up to $1000 \mathrm{~K}$ power $\approx 10 \mathrm{~mW}$ is needed. Taking into account that the wire resistance $\leq 1 \Omega$, one can conclude that current of the order of a few hundred $\mathrm{mA}$ is needed for heating modeling by dc through the wire.

Figure 2 shows the calculated values of power released on the wires of diameters 10 and $30 \mu \mathrm{m}$ at scattering on them of a beam with average current $0.9 \mathrm{~mA}$ depending on relative distance of the wire from the center of the beam with horizontal transverse size $\sigma_{x}=3 \mathrm{~mm}$. The value of ionization losses $\Delta E / \Delta t$ is set equal to $5.7 \times$ $10^{-11} \mathrm{~J} / \mathrm{m}$ [5]. The current distribution was supposed Gaussian in the beam cross section. Characteristic values of the beam energy released on the wire are a few $\mathrm{mW}$.

The equilibrium temperature $T_{\mathrm{eq}}$ of the wire is determined from the equation of the balance

$$
W_{1}+W_{2}=\Delta E / \Delta t \text {. }
$$

Solutions of this equation with respect to $T_{\mathrm{eq}}$ at different distances of wire from the beam center are presented in Fig. 3. One can see that for wires of diameters 10 and $30 \mu \mathrm{m}$ the maximum temperatures are reached when the wire is close to the beam center and are equal to 1100 and $850 \mathrm{~K}$, respectively.

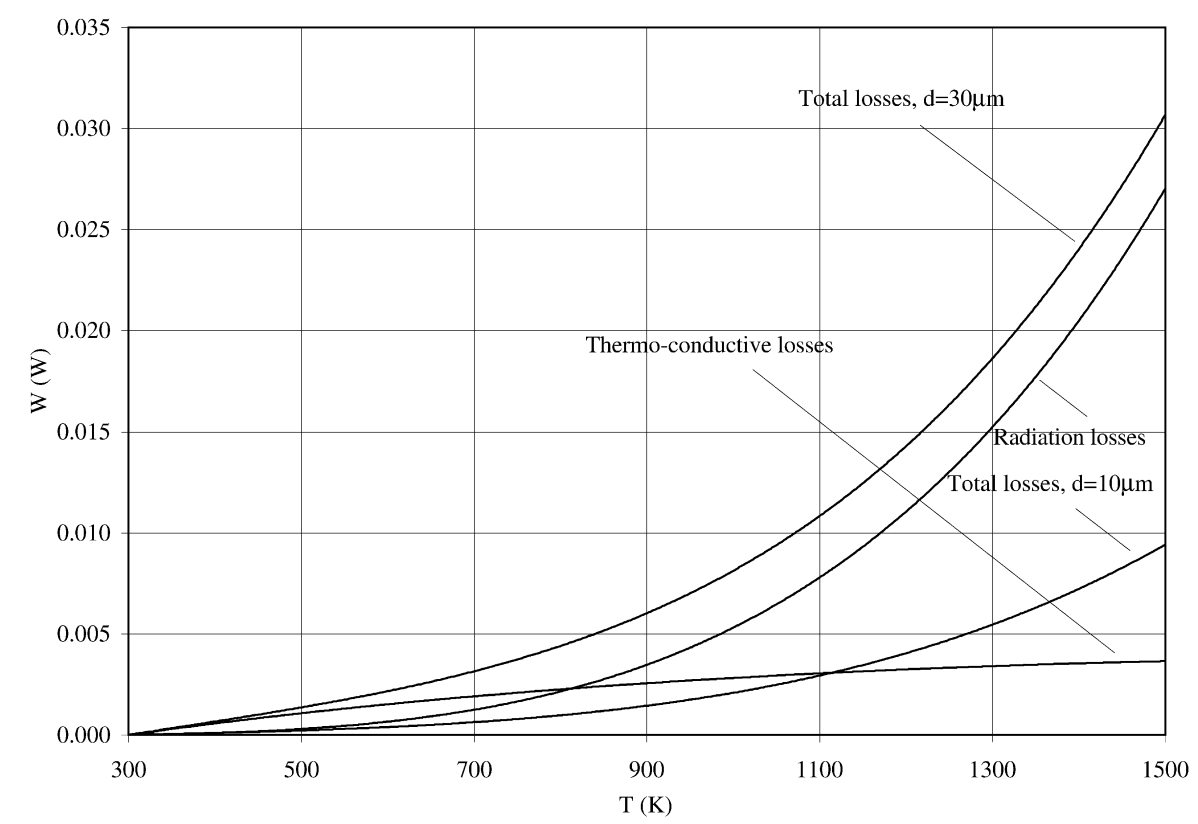

FIG. 1. Total energy losses for tungsten wire, $d_{1}=30 \mu \mathrm{m}, d_{2}=10 \mu \mathrm{m}, l=20 \mathrm{~mm}, \sigma_{x}=3 \mathrm{~mm}, \sigma_{z}=1 \mathrm{~mm}$. For the $d_{1}=30 \mu \mathrm{m}$ case, radiation losses and thermoconductive losses are also presented. 


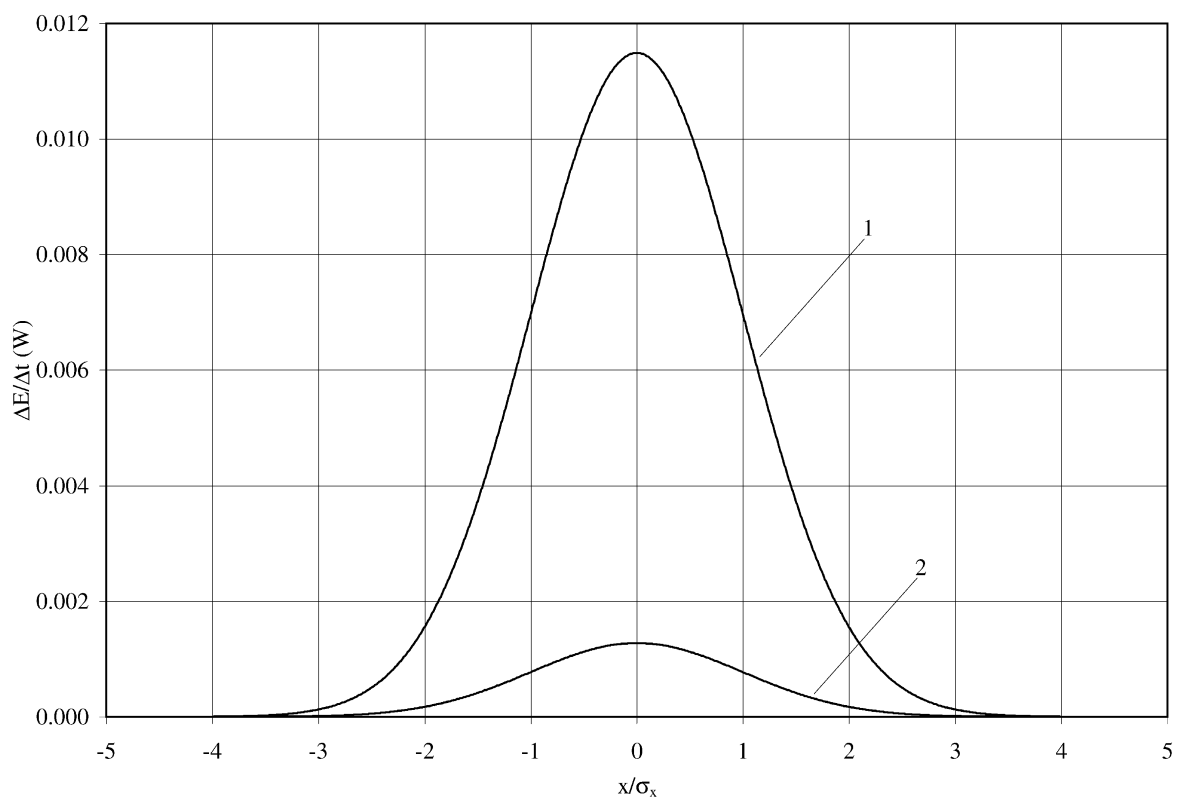

FIG. 2. Heat power from bunch scattering on wire, $I=0.9 \mathrm{~mA}, d_{1}=30 \mu \mathrm{m}, d_{2}=10 \mu \mathrm{m}$.

The time of the wire thermalization $\tau$ is also an important parameter for the scanning. To estimate the total time of the beam scattering one can use the formula

$$
\tau \cong E_{\text {store }} / W
$$

where $E_{\text {store }}$ is the thermal energy stored in the wire $\left[E_{\text {store }}=\left(\pi d^{2} / 4\right) \sigma_{y} c_{V}\left(T-T_{0}\right)\right.$, where $c_{V}$ is the heat capacity per unit volume of the wire material; for tungsten $\left.c_{V}=2.86 \times 10^{6} \mathrm{~J} \mathrm{~m}^{-3} \mathrm{~K}^{-1}\right]$. Figure 4 represents calculated values of thermalization times for tungsten wires of diameters 10 and $30 \mu \mathrm{m}$. As is seen from the graphics, the thermalization time is minimal in the beam center and is 2 and $3 \mathrm{~s}$, respectively. In the beam periphery (at the distances $2 \sigma_{x}$ ) this time is approximately doubled.

An estimate of transverse force for relativistic beams acting on wire gives values of the order of $W / c \sim$ $10^{-11} \mathrm{~N}$ ( $c$ is the speed of light). This force is negligibly small compared with the wire heating effects.

\section{MODEL EXPERIMENT}

Influence of the wire temperature on its normal oscillation frequency $f$ is determined by its elongation $\Delta l$ :

$$
\Delta f / f=-(1 / 2) \Delta l / l=\alpha_{s} \Delta T,
$$

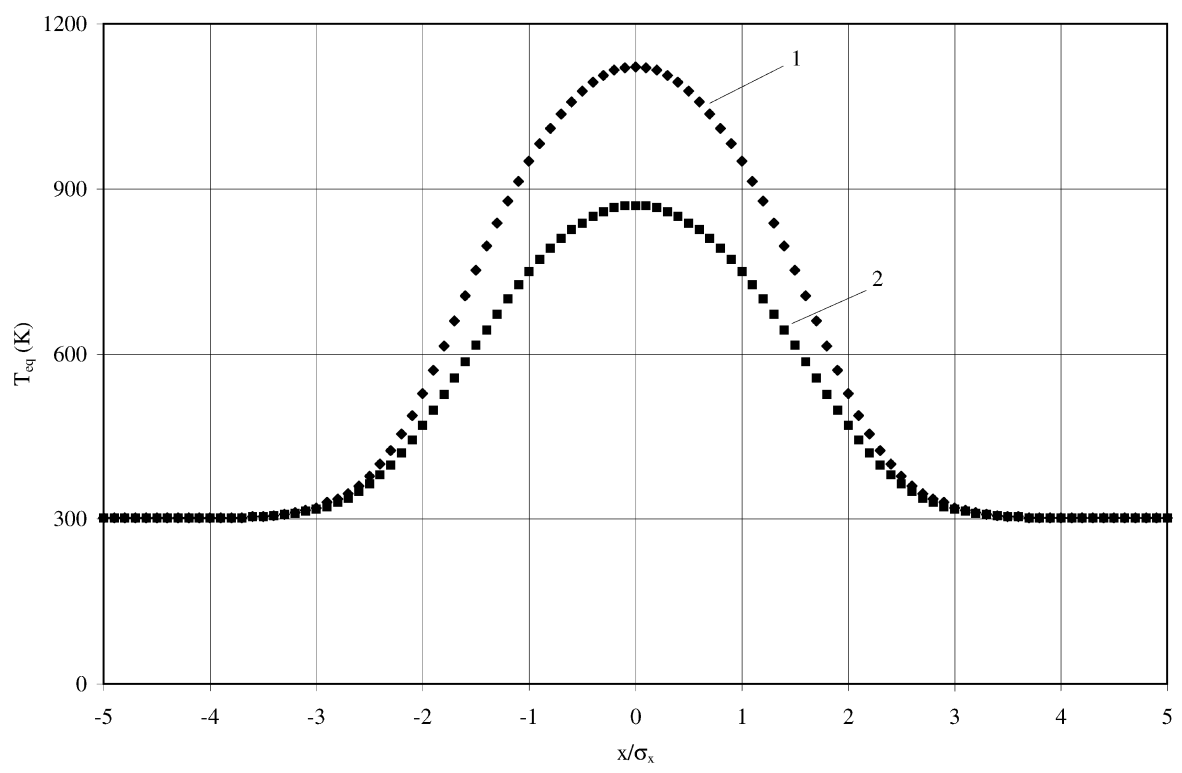

FIG. 3. Wire equilibrium temperature $T_{\mathrm{eq}}$ at different distances of the wire from beam center, $d_{1}=30 \mu \mathrm{m}, d_{2}=10 \mu \mathrm{m}$. 


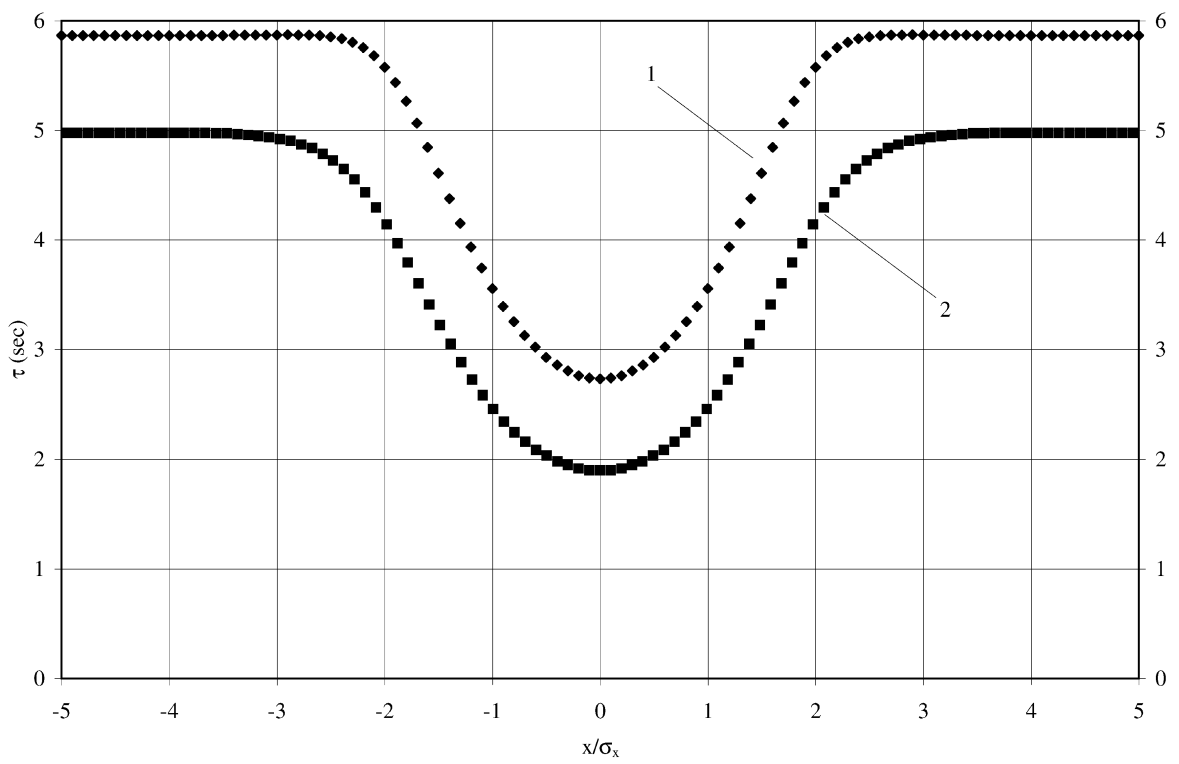

FIG. 4. Wire thermalization time $\tau$ depending on distance from beam center, $d_{1}=30 \mu \mathrm{m}, d_{2}=10 \mu \mathrm{m}$.

where $\Delta T$ is the temperature variation. Coefficient $\alpha_{s}$ for metals varies from a few units of $10^{-6} \mathrm{~K}^{-1}$ (tungsten, molybdenum) up to $10^{-5} \mathrm{~K}^{-1}$ (steels, bronzes, brasses, aluminum alloys). Some special alloys have an extra low temperature coefficient of thermal expansion, e.g., for invar $\alpha_{s}=(0.01-2.0) \times 10^{-6} \mathrm{~K}^{-1}[15]$.

For the rigidly fixed wire ends, more essential is the influence of temperature changes on the wire tension arising from the inequality of the coefficients of thermal expansion of the wire $\alpha_{s}$ and the base $\alpha_{b}$. Thus [16]

$$
\frac{\Delta f}{f}-\frac{1}{2} \frac{\Delta F}{F} \approx \frac{E S}{F}\left(\alpha_{b}-\alpha_{s}\right) \Delta T
$$

where $E$ is Young's modulus. At deducing (7) it is taken into account that $\Delta F / F=E \Delta l / l$. In Eq. (7) a large dimensionless factor $E S / F$ is separated out. For the tungsten wires of diameter $\sim 100 \mu \mathrm{m}$, the upper limit of loads for stable frequency generation is less than $10 \mathrm{~N}$. In this case $E S / F>400$ for the beryl bronze wires of diameter $90 \mu \mathrm{m}$ and $F \sim 3 \mathrm{~N}$ near the upper limit of the operating range of loads $E S / F \sim 300$. Thus, for the rigidly fixed wire the dominating factor is the influence of the temperature on the wire tension.

Heating of the scanning wire was modeled by alternating and direct currents through the wire. Wire normal oscillations were generated in two cases: with one free end

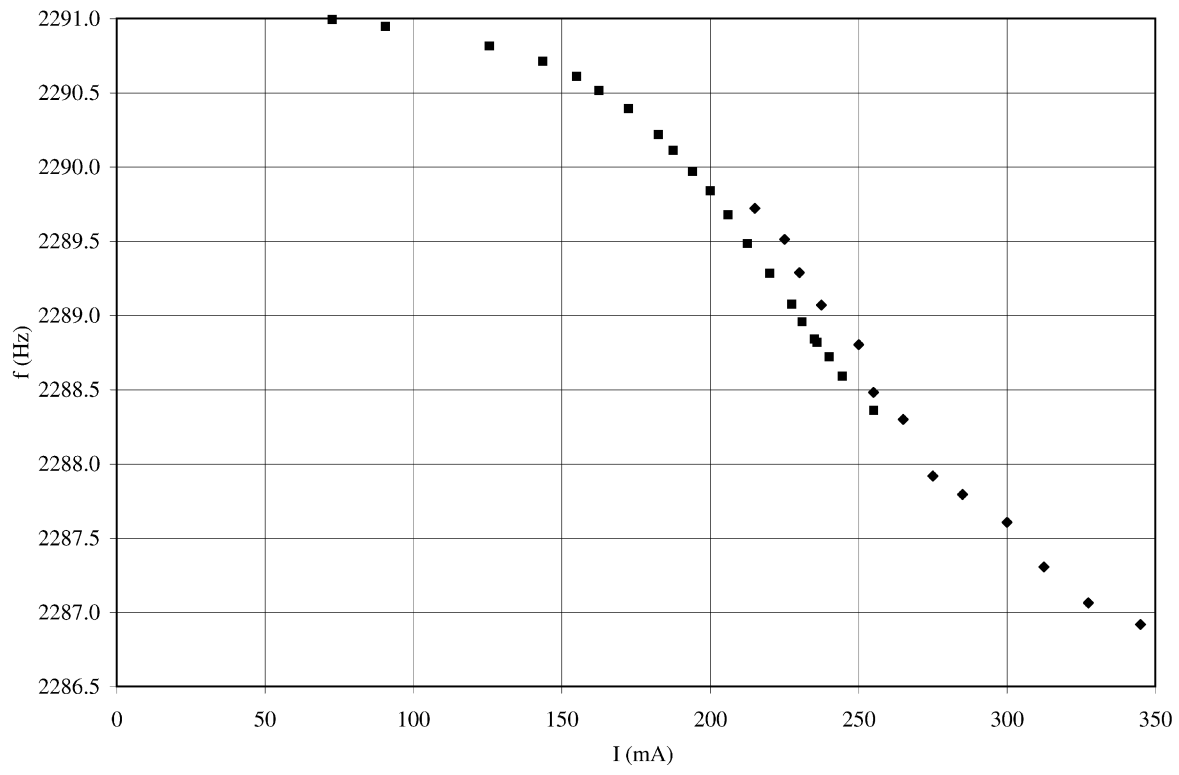

FIG. 5. Wire oscillation frequency dependence on the current through the wire in the model experiment. 


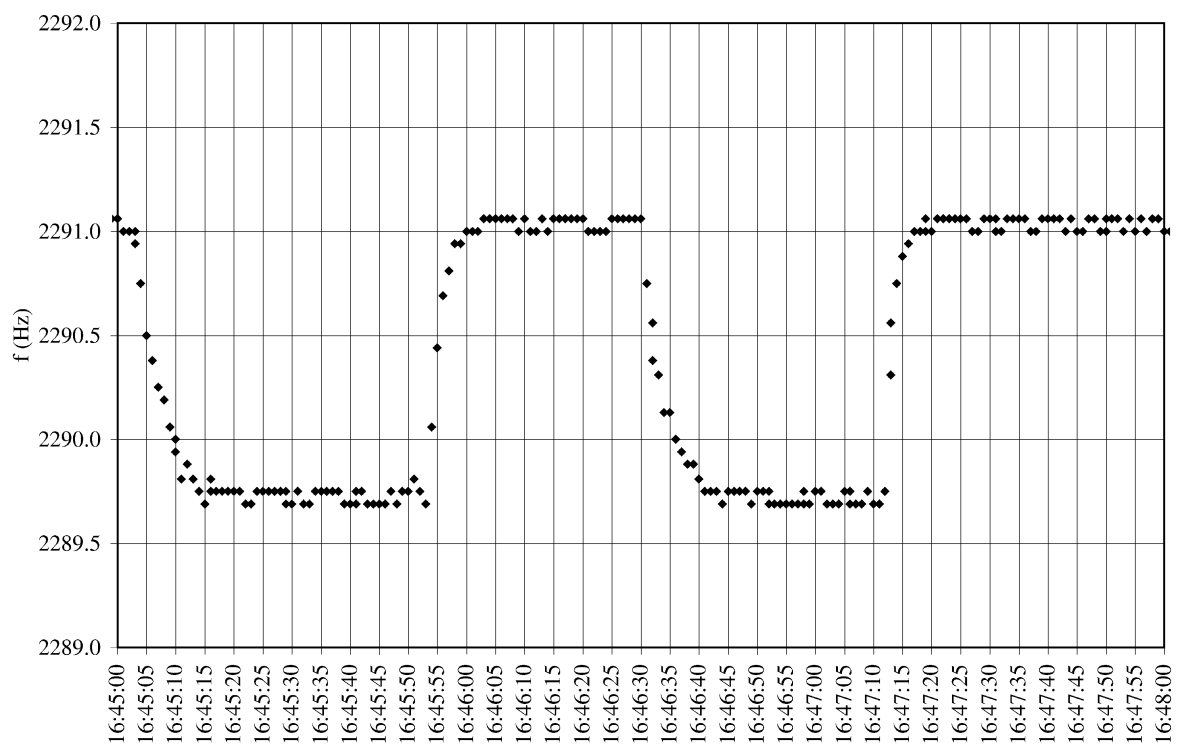

FIG. 6. Transient characteristics of the wire oscillation frequency at switching on/off current $I=215 \mathrm{~mA}$ through the wire. One end of the wire is free.

and with fixed ends of the wire. The experiments were done in forevacuum evacuation.

In experiments with alternating current, forced wire oscillations of current frequency arise (at the current frequency $50 \mathrm{~Hz}$ normal oscillations broke at the current $l>50 \mathrm{~mA}$ ). When heating by direct current, the same tungsten wires of diameter $70 \mu \mathrm{m}$ were used.

Data obtained at constant wire tension (one free end of the wire) are presented in Fig. 5. Results of two series of measurements are shown. The first series (squares) was carried out for currents from 70 to $250 \mathrm{~mA}$; glow appeared at $235 \mathrm{~mA}$. Later on an experiment with currents from 215 to $345 \mathrm{~mA}$ was done (diamonds). In the first series the current was changed stepwise, increasing consequently from the lower values to the upper ones. In the second case between two consecutive values the current was nullified, each current value being held during 2 min. In the second series the frequency was well restored at each switching on for currents up to $345 \mathrm{~mA}$. At upper values of the current after each heating cycle the frequency systematically went up. Yellow glow $(T \approx$ $1000 \mathrm{~K}$ ) appears at currents more than $300 \mathrm{~mA}$.

Figure 6 shows the transient characteristics of the frequency of the wire with one free end at switching on/off

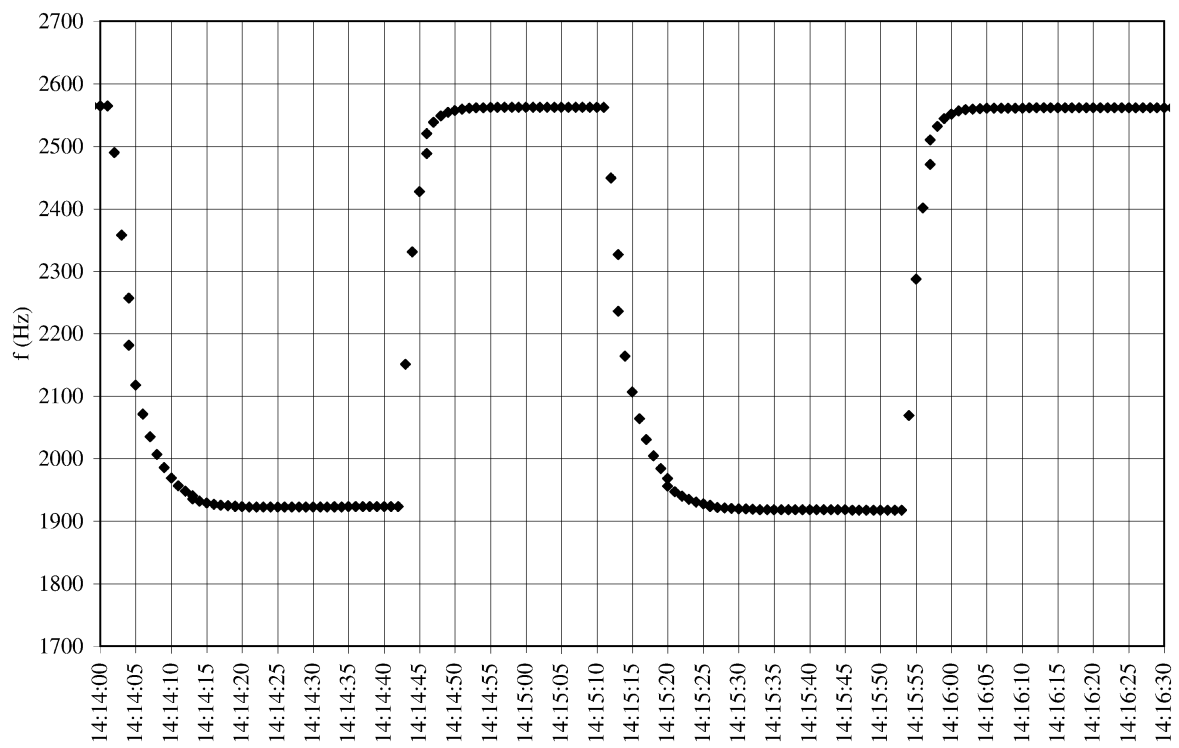

FIG. 7. Transient characteristics of the wire oscillation frequency at switching on/off current $I=215 \mathrm{~mA}$ through the wire. Both ends of the wire are fixed. 
current $215 \mathrm{~mA}$. It is seen that the frequency exceeds its steady state value within a few seconds. This value can be used to estimate the thermalization time $\tau$.

Similar experiments were done for wires with fixed ends. As mentioned above, the frequency change interval was much wider. Figure 7 shows the transient characteristics of the wire frequency at switching on/off current $215 \mathrm{~mA}$.

Restoration of the frequency in both cases (Figs. 6 and 7) was good enough; i.e., heating of the wire up to $1000 \mathrm{~K}$ does not lead to irreversible changes of wire parameters.

Thus, the model experiments show that reproducible generation of wire normal oscillations is possible when it is considerably heated. Theoretical estimates are in accord with obtained experimental data.

\section{WIRE UNDER BUNCH-PROPOSAL FOR EXPERIMENT}

Let us discuss some specific problems that can arise at scanning of the accelerator beam by a vibrating wire.

\section{A. Magnetic field}

There are two possibilities in the scheme of wire oscillation generation: use a permanent magnet or use the accelerator magnetic fields (dipole magnets, quadrupole lenses).

In pickups developed by the authors, normal oscillations of the wire were excited in an autogeneration scheme. Exciting action on the wire arises as a result of interaction between the current through the wire and magnetic field of the samarium cobalt magnet. The field is localized in the magnet operating gap of length $10 \mathrm{~mm}$. The characteristic field inside the gap is about $8 \mathrm{kG}$.

To keep the transverse effective square of the wire with respect to the ongoing bunch, separation of oscillations along the bunch axis is desirable. Accelerator magnetic fields meet this requirement. It can also be satisfied when embedded small magnets are used.

Fields of dipole magnets are strong enough for oscillation generation; however, they fix the horizontal movement of the wire.

It is also possible to use the fields of quadrupole lenses. However, in such fields even modes of the wire normal oscillations are possible. Scanning can be done in vertical, horizontal, or under the angle $45^{\circ}$ direction of the wire.

Use of the vibrating wire scanners with their own magnets located in accelerator free spaces is also possible. In this case it is preferable to intersect the bunch at $1 / 4$ of the wire length and magnetic circuit located symmetrically with respect to the wire center. In such a way, generation of the second harmonic will be provided. The magnetic field outside the gap is to be carefully screened.

To demonstrate the possibility of generation of different harmonics of the wire oscillations we have carried out an experiment with shortened magnetic circuit shifted along the wire. Results of the experiment are presented in Fig. 8. The horizontal axis is the shift of the center of the magnetic circuit with respect to a fixed end of the wire. Position $20 \mathrm{~mm}$ corresponds to the wire center. The shift of the magnetic circuit downward (in Fig. 8, to

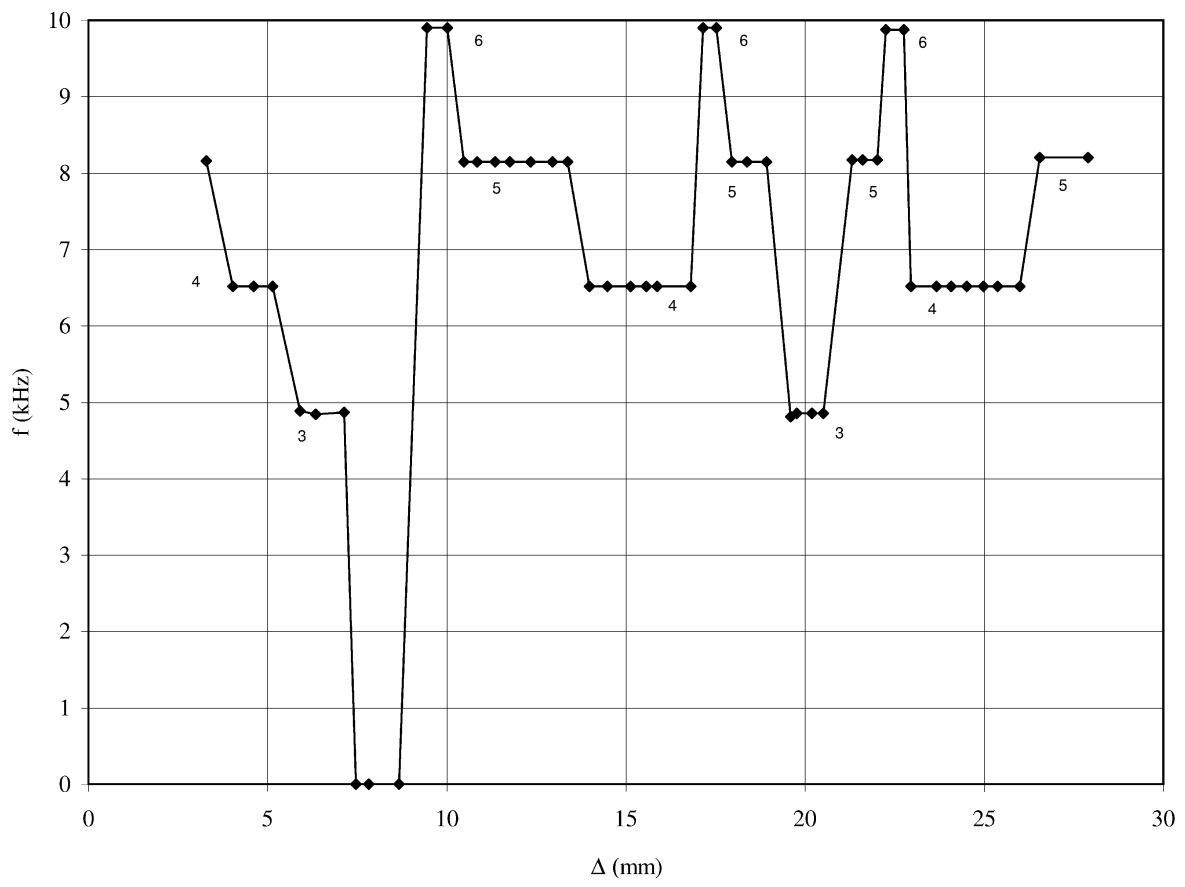

FIG. 8. Higher harmonics generation depending on magnet shift $\Delta$ from the upper end of the wire. The value $\Delta=20 \mathrm{~mm}$ corresponds to the wire center. 
the right) was limited by the sensor's construction; however, one can see that excitation of normal harmonics of the wire is symmetric with respect to the shifts from the wire center. Usage of a short magnetic circuit results in the generation of a third harmonic in the central position. By shifting of the magnetic circuit one can obtain generation of third, fourth, fifth, or sixth harmonics (indicated by numbers in the figure). There was a silence zone too. Of course, results of the experiments depend on geometric characteristics; however, they show the possibility of stable generation of the needed harmonic.

Self-magnetic fields of the beam are far less than the external accelerator fields and their influence on the wire oscillations frequency is negligibly small.

\section{B. Radiation resistance}

Tungsten and bronze (beryl bronze) are used in both scanning wire and vibrating wire technologies. In both cases they have high radiation resistance. The electronic circuit exciting the wire scanner oscillations is located far from the wire and can be sufficiently screened from radiation. Other parts of the vibrating wire scanner are passive elements and are also radiation resistant.

\section{Thermal stability, thermalization times}

Stability with respect to wire heating is one of the main problems in developed wire scanner technologies. To solve this problem, the scanning was done at a high speed, up to $\approx 10 \mathrm{~m} / \mathrm{s}[3,4]$; i.e., the wire passes across the millimeter size bunch during $10^{-4} \mathrm{~s}$. Such intervals are too small for thermalization of the wire. Note that the estimate (5) of $\tau$ can be lowered, because real movement from one point to another will result in smaller temperature jumps. However, one cannot expect thermalization times less than $10^{-2} \mathrm{~s}$ at temperatures where long-time exploitation of wire is possible. Usage of thin and short wires will give some advantage. It is possible that the problem of the limit on thermalization time can be solved by improvement of the wire ends fixing system, which now is essentially simplified in comparison with similar wire scanning systems.

\section{Vacuum system}

Since the vibrating scanner system consists of one unit, its installation into an accelerator vacuum system will encounter fewer problems than in the case of a twounit wire scanner system. It is also desirable to have a system of wire replacement without loss of vacuum in the acceleration chamber.

\section{E. Resolution, measurement accuracy, calibration method}

Resolution and measurement accuracy of the proposed method are primarily determined by resolution and accu- racy of used vibrating wire sensors. We measured the frequency by a simple method consisting of measurements of entire periods of oscillations during the measurement time $g$. The frequency was preliminarily multiplied by factor $k$, which was selected from the series $16,32,64,128$. Thus, at $k=128$ the resolution of frequency is $\sim 7 \mathrm{mHz}$ at frequency $\sim 3 \mathrm{kHz}$ with their ratio $\sim 2.6 \times 10^{-6}$. Note that the relative root-mean-square deviation of frequency in longtime experiment in steady conditions was $\sim 6 \times 10^{-6}$, which is close to the sensor's resolution. In the region of "hot" string, where $T \gg T_{0}$ and $W_{2}$ in Eq. (4) is negligible, the relationship between the relative change of frequency with that of falling particles is determined by the expression

$$
\frac{\Delta p}{p(x)}=\frac{4 F}{E S T\left(\alpha_{b}-\alpha_{s}\right)} \frac{\Delta f}{f} .
$$

For a tungsten string with $E S / F \sim 400, T \sim 1000 \mathrm{~K}$, $\alpha_{b}-\alpha_{s} \sim 10^{-5} \mathrm{~K}^{-1}$, we obtain $\Delta p / p(x) \sim \Delta f / f$. Thus, the relative resolution of falling particles is equal to the resolution of the wire sensor frequency.

The question of measurement accuracy is more complicated and requires a reasonable compromise between the speed of scanning and the sensor's frequency measurement taking into account the characteristic times of string thermalization. As seen from Fig. 4, the total scanning time lasts $\sim 1 \mathrm{~s}$. At the signal's frequency measurement interval $\sim 10 \mathrm{~ms}$, the relative accuracy of a single measurement will be $\sim 10^{-4}$. Note that the equilibrium temperatures of the string in adjacent positions differs by $10 \mathrm{~K}$.

Presented values are to be compared with traditional methods of slow wire scanning. In Ref. [1] with beam scanning step $0.7 \mathrm{~mm}$ with delay in each point $\sim 4.9 \mathrm{~s}$ (number of points 135) and signal-to-noise ratio $\sim 100$, the relative resolution of the method is estimated to be $3 \%$. Note that the resolution depends on the information source ( $\gamma$ photons or secondary electrons).

The calibration of the proposed method can be done by referring to the total current of the beam, measured in another way, with the integral of the beam profile obtained from beam scanning by vibrating wire. Application of the proposed method is reasonable to begin from a traditional scanning system replacing the "dead" wire by the vibrating one. Comparison of these methods will give valuable information on calibration.

\section{CONCLUSION}

An indubitable advantage of the proposed method is the compactness of the whole scanning system and the elimination of the unit of radiation detectors.

Also note that the proposed method can be used for peripheral monitoring far from the equilibrium trajectory of the beam. In this case rather large intervals of frequency measurement can be used. At $g=1 \mathrm{~s}$ one can observe less than $10^{-5}$ parts of the total flux of the beam. 
Such measurements are very important for problems of beam emittance growth and halo formation.

To overcome the problems of wake field heating by sharp bunch structure of the beam, the sensor can be prepared from two wires-one metallic as a vibrating wire and the second dielectric as a target wire.

\section{ACKNOWLEDGMENTS}

This work was supported by HTM Reetz GmbH (Berlin). The authors would like to thank R. Reetz, A. Ts. Amatuni, and the participants of the HERA accelerator physics seminar (DESY) for encouraging discussions and helpful support.

[1] T. Suwada, N. Iida, Y. Funakoshi, T. Kawamoto, and M. Kikuchi, KEK Report No. 97-184, 1997.

[2] M. Martini and H. O. Schoenauer, CERN Report No. PS97-018-CA, 1997.

[3] Ch. Steinbach, CERN Report No. PS-95-04-OP, 1995.

[4] V. Agoritsas, E. Falk, F. Hoekemeijer, J. Olsfors, and C. Steinbach, CERN Report No. PS-95-06-BD/OP, 1995.

[5] J. Bosser et al., Nucl. Instrum. Methods Phys. Res.,
Sect. A 234, 475 (1985).

[6] R. Tatchan, in Proceedings of the Particle Accelerator Conference, Vancouver, Canada, 1997 (IEEE, Piscataway, NJ, 1998).

[7] R. Fulton, SLAC Report No. PUB-4605, 1988.

[8] C. Field, SLAC Report No. PUB-6717, 1994.

[9] D. McCormick et al., SLAC Report No. PUB-6615, 1994.

[10] Methods of Experimental Physics (Academic, New York, London, 1959), Vol. 1.

[11] Ju.F. El'tsev et al., in Trudy FIAN (FIAN, Moscow, 1984), Vol. 150.

[12] J. Billan, in Proceedings of the CERN Accelerator School, Montreux, Switzerland, 1992, edited by S. Turner (CERN, Geneva, 1992), p. 17.

[13] S. G. Arutunian, I.E. Vasinyuk, N. M. Dobrovolsky, S. L. Egiazarian, M. R. Mailian, I. G. Sinenko, and A. V. Sinyavskiy (to be published).

[14] S. G. Arutunian, N.M. Dobrovolsky, S.L. Egiazarian, M. R. Mailian, I. G. Sinenko, A. V. Sinyavski, and I.E. Vasinyuk, in Proceedings of the 1999 Particle Accelerator Conference, New York (to be published), p. 1468.

[15] I. S. Grigor'ev and E.Z. Mejlikhov, in Physical Data Handbook (Energoatomizdat, Moscow, 1991).

[16] D. I. Agejkin et al., in Control and Regulation Pickups (Mashinostroenie, Moscow, 1965). 